\title{
Plasmonic Antennas with Electric, Magnetic, and Electromagnetic Hot Spots Based on Babinet's Principle
}

\author{
Martin Hrtoň $\odot,{ }^{1}$ Andrea Konečná $\odot,{ }^{2}$ Michal Horák $\odot,{ }^{1}$ Tomáš Šikola $\odot,{ }^{1,3}$ and Vlastimil Křápek ${ }^{1,3, *}$ \\ ${ }^{1}$ Central European Institute of Technology, Brno University of Technology, Purkyňova 123, Brno 61200 , \\ Czech Republic \\ ${ }^{2}$ Materials Physics Center CSIC-UPV/EHU, Paseo Manuel de Lardizabal 5, San Sebastián 20018, Spain \\ ${ }^{3}$ Institute of Physical Engineering, Brno University of Technology, Technická 2, Brno 616 69, Czech Republic
}

(Received 11 October 2019; revised manuscript received 10 March 2020; accepted 28 April 2020; published 19 May 2020)

\begin{abstract}
We theoretically study plasmonic antennas featuring areas of extremely concentrated electric or magnetic field, known as hot spots. We combine two types of electric-magnetic complementarity to increase the degree of freedom for the design of the antennas: bowtie and diabolo duality and Babinet's principle. We evaluate the figures of merit for different plasmon-enhanced optical spectroscopy methods and optical trapping: field enhancement, decay rate enhancement, quality factor of the plasmon resonances, and trapping potential depth. The role of Babinet's principle in interchanging electric and magnetic field hot spots and its consequences for practical antenna design are discussed. In particular, diabolo antennas exhibit slightly better performance than bowties in terms of larger field enhancement and larger $Q$ factor. For specific resonance frequency, diabolo antennas are considerably smaller than bowties, which makes them favorable for the integration into more complex devices but also makes their fabrication more demanding in terms of spatial resolution. Finally, we propose a Babinet-type dimer antenna featuring electromagnetic hot spot with both the electric and magnetic field components treated on an equal footing.
\end{abstract}

DOI: 10.1103/PhysRevApplied.13.054045

\section{INTRODUCTION}

Plasmonic antennas (PAs) are metallic particles widely studied for their ability to control, enhance, and concentrate electromagnetic field [1]. Strikingly, the field in the vicinity of plasmonic antennas, the so-called near field, can be focused into a deeply subwavelength region. At the same time, the field is strongly enhanced with respect to the driving field, which can be, e.g., a plane wave. Focusing of the field stems from the excitation of localized surface plasmons (LSP) - quantized oscillations of the free electron gas in the metal coupled to the evanescent electromagnetic wave propagating along the boundary of the metal.

In judiciously designed plasmonic antennas, local spots of particularly enhanced electric or magnetic field can be formed, referred to as hot spots. The hot spots typically arise from the interaction between adjacent parts of a plasmonic antenna separated by a small gap $[2,3]$ but they can be also based on the lightning rod effect (a concentration of the field at sharp features of the antenna) [4-6] or combination of both. In various studies, electric hot spots have been reported over a broad spectral range from $\mathrm{THz}$ [5] (hot spot size $\lambda / 60000$ predicted from electromagnetic simulations,

\footnotetext{
*vlastimil.krapek@ceitec.vutbr.cz
}

with $\lambda$ denoting the wavelength of the incident wave) to visible [7] (hot spot size $\lambda / 600$ and enhancement $>500$ ).

Depending on the enhanced field, hot spots can be classified as electric, magnetic, or electromagnetic. A variety of plasmonic antennas with specific shapes, sizes, and materials exists for both electric and magnetic hot spots. Electric hot spots have been observed in the nanorod dimer antennas, bowtie antennas [8], or chains of plasmonic nanoparticles [2,9]. Magnetic hot spots are formed in diabolo antennas [10], nanorings [11], or split-ring resonators [12]. Electromagnetic hot spots with simultaneous enhancement of both electric and magnetic field are unique for plasmonic antennas [13]. Their formation has been observed in dielectric resonators (silicon nanodimers) [14].

Hot spots can be involved in many applications including surface-enhanced Raman scattering [2,15,16], more efficient photocatalysis [17], or fluorescence of individual molecules [18]. Metallic resonators with enhanced magnetic field (magnetic hot spots) are used to increase the efficiency of magnetic spectroscopies such as electron paramagnetic resonance [19]. Electromagnetic hot spots can be useful for studies of materials with combined electric and magnetic transitions such as rare earth ions [20,21]. Combined enhancement of electric and magnetic field finds applications also in optical trapping [22], metamaterials [23], or nonlinear optics [24]. 
For experimental characterization of plasmonic hot spots, the available methods are, e.g., scanning near-field optical microscopy [14,25-27], photon-scanning tunneling microscopy [3], two-photon luminescence spectroscopy [28], or photothermal-induced resonance [29].

Bowtie geometry of plasmonic antennas features a particularly strong electric hot spot. Bowtie antennas are planar antennas consisting of two metallic triangular prisms (wings) whose adjacent apexes are separated by a subwavelength insulating gap. The hot spot arises from the interaction between the apexes combined with the lightning rod effect (the charge of LSP accumulates at the apexes). When the insulating gap is replaced with a conductive bridge, a diabolo plasmonic antenna is formed. Instead of charge accumulation, electric current is funneled through the bridge, resulting in a magnetic hot spot. Both the bowtie and diabolo antennas have been frequently studied [8,10,18,30-35].

Various optimization and modification approaches have been proposed with the aim to enhance the properties of the bowtie and diabolo antennas, including the gap optimization [30], fractal geometry [36], or Babinet's principle. Babinet's principle relates the optical response of a (direct) planar antenna and an inverted planar antenna with interchanged conductive and insulating parts. Both the direct and inverted antennas shall support LSP with identical energies, but with interchanged electric and magnetic near field [37,38]. Consequently, when the direct antenna features an electric hot spot, the inverted antenna features a magnetic hot spot and vice versa. The validity of Babinet's principle for the plasmonic antennas has been experimentally verified $[39,40]$, although some quantitative limitations have been found in particular in the visible spectral range [41,42].

A unique combination of Babinet's complementarity and bowtie and diabolo duality extends a degree of freedom for the design of plasmonic antennas featuring hot spots. In our contribution we compare the two antennas featuring an electric hot spot (bowtie and inverted diabolo) and the other two featuring a magnetic hot spot (diabolo and inverted bowtie). By electromagnetic modeling we retrieve the characteristics of the hot spots and figures of merit of relevant plasmon-enhanced optical spectroscopy methods. Finally, we design Babinet dimer antennas featuring electromagnetic hot spots.

\section{METHODS}

In all simulations, the bowtie and diabolo antennas are represented by two gold triangles or triangular apertures (as shown in Fig. 1) of $30 \mathrm{~nm}$ height on a semiinfinite glass substrate. Babinet dimers are formed by two complementary PAs (direct and inverted, each of 30 $\mathrm{nm}$ height) vertically separated by a 10 -nm-thick layer with the refractive index equal to 1.5 corresponding to
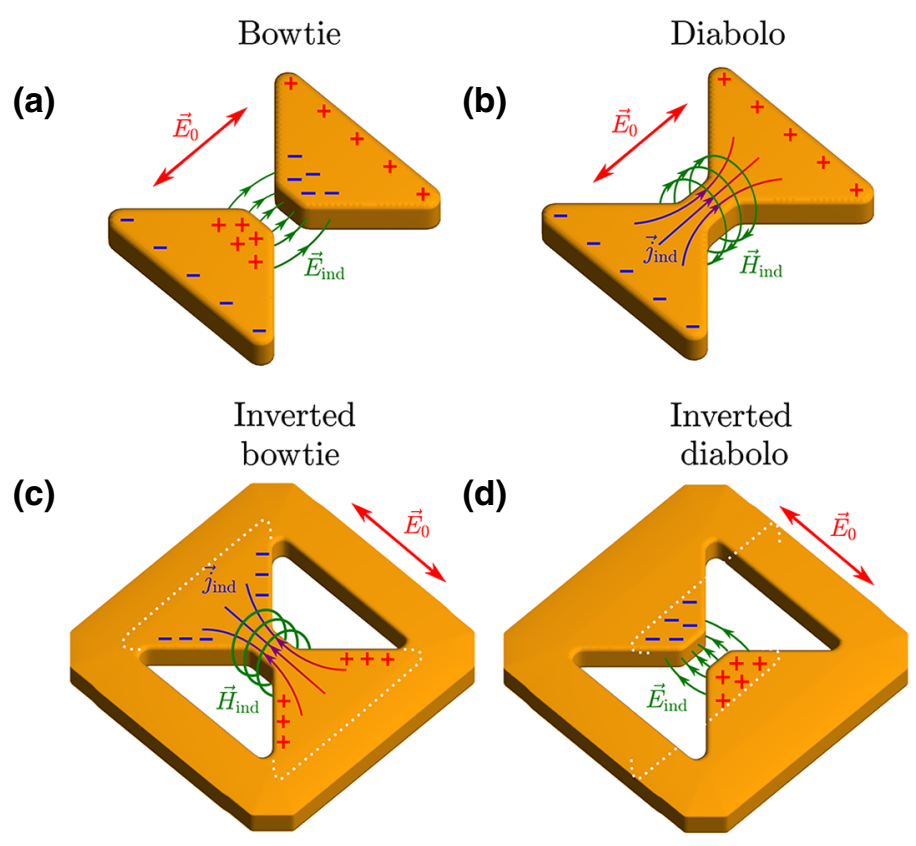

(e)

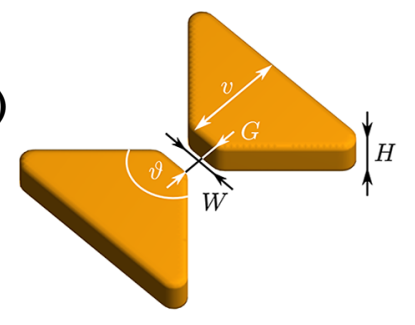

FIG. 1. Schemes of four plasmonic antennas featuring hot spots: (a) bowtie, (b) diabolo, (c) inverted bowtie, (d) inverted diabolo. Metallic and hollow parts are represented by golden and white color, respectively. Driving electric field is indicated by red arrows. Charge or current accumulation and formation of the electric or magnetic hot spot are shown as well. The dotted white line in (c) indicates the qualitative correspondence between the diabolo and inverted bowtie antennas, while the dotted white line in (d) indicates a similar correspondence between the bowtie and inverted diabolo antennas. Dimension parameters of the antennas are shown in (e).

poly(methyl methacrylate). The whole dimer lies on a semi-infinite glass substrate. The dielectric function of gold is taken from Ref. [43] and the refractive index of the glass is set equal to 1.47.

The electromagnetic field is calculated with the finite difference in time domain (FDTD) method using a commercial software Lumerical.

Scattering efficiencies and the near-field distribution are calculated using a plane wave as illumination. Transition decay rates are calculated as the decay rate of the power radiated by an oscillating electric or magnetic dipole into its surrounding (total decay rate) and into far field (radiative decay rate). The dipole is positioned at the vertical symmetry axis of the antenna. The decay rates are averaged over three orthogonal polarizations of the dipole. 


\section{RESULTS AND DISCUSSION}

\section{A. Plasmonic antennas, modes, and hot spots}

Plasmonic antennas involved in the study and their operational principle are illustrated in Fig. 1. The bowtie antenna consists of two disjoint triangular gold prisms. An oscillating electric field applied along the long axis of the antenna drives the oscillations of charge that is funneled by the wings of the antenna and accumulated at the adjacent tips [Fig. 1(a)]. Combined effects of plasmonic field confinement, charge funneling, and charge concentration (lightning rod effect) give rise to an exceptionally high field in the area between the triangles, by orders of magnitude higher than the driving field. In the diabolo antenna, the triangles are connected with a conductive bridge, through which a concentrated current flows instead of charge accumulation [Fig. 1(b)]. A magnetic hot spot is formed around the bridge. The inverted bowtie antenna is formed by two disjoint triangular apertures in an otherwise continuous gold film. Babinet's principle predicts that for a complementary illumination (i.e., transverse oscillating electric field) a complementary magnetic hot spot is formed. This can be understood also intuitively as the antenna resembles a rotated diabolo antenna [see dotted line in Fig. 1(c)]. Finally, the inverted diabolo antenna, which on the other hand resembles the bowtie antenna, features an electric hot spot Fig. 1(d).

The dimension parameters of the antennas are schematically depicted in Fig. 1(d). The thickness of the gold film is set to $H=30 \mathrm{~nm}$. The size of the right isosceles triangles (i.e., $\vartheta=90^{\circ}$ ) is described by the wing length $v$. The opposite triangles share a common apex. The isolating gap in bowtie antennas and the conductive bridge in diabolo antennas have the length $G$ equal to the width $W$. These dimensions do not scale with the size of the antenna (the only scalable parameter is thus $v$ ) and are set to $30 \mathrm{~nm}$ to reflect a convenient resolution of fabrication methods such as electron-beam lithography and focused ion-beam lithography. In general, one could expect stronger hot spots for narrower gaps or bridges due to stronger charge or current concentration. All edges are rounded with a radius of $10 \mathrm{~nm}$. The antennas are placed on a semi-infinite glass substrate (refractive index 1.47). The dielectric function of gold is taken from Johnson and Christy [43].

One of the quantities characterizing plasmonic response of antennas is their scattering efficiency $Q_{\text {scat }}$. It describes the power $P_{\text {scat }}$ scattered by the antenna illuminated with a monochromatic plane wave with an intensity $I_{0}$ and is defined as $Q_{\text {scat }}=P_{\text {scat }} /\left(I_{0} S\right)$, where $S$ denotes the geometrical cross section of the antenna. Spectral dependencies of $Q_{\text {scat }}$ for all four PA types are shown in Fig. 2 and the energies of the lowest scattering peak corresponding to a dipole plasmonic mode are shown in Fig. 3. We observe that Babinet's principle holds reasonably well. The peak energies of the scattering efficiency in the complementary PA (i.e., bowtie and inverted bowtie, diabolo and inverted diabolo) of the same size differ by less than $11 \%$. The difference is less pronounced for large antennas, in line with the requirements of Babinet's principle: perfectly thin and opaque metal [42]. For the bowtie geometry, the scattering peaks of inverted PAs are less intense and redshifted with respect to direct PAs (as is the case also for disc-shaped antennas [42]), while opposite is true for the diabolo geometry.

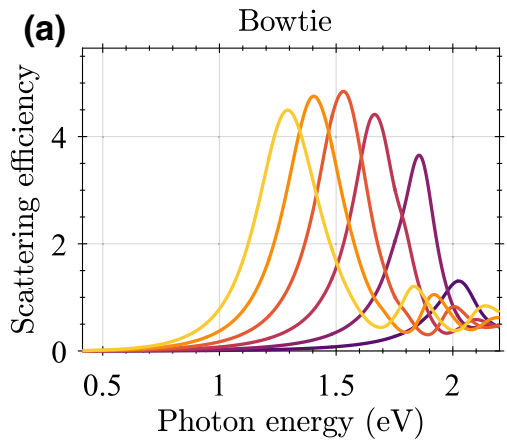

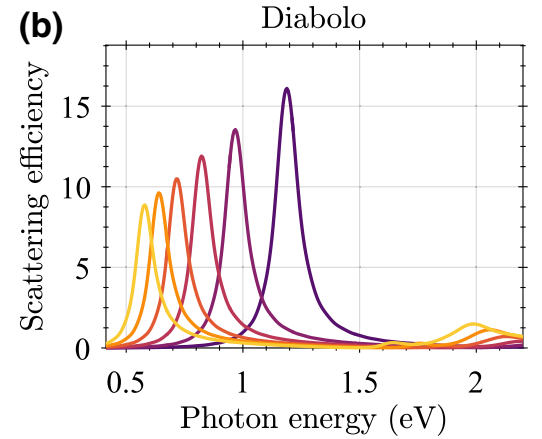

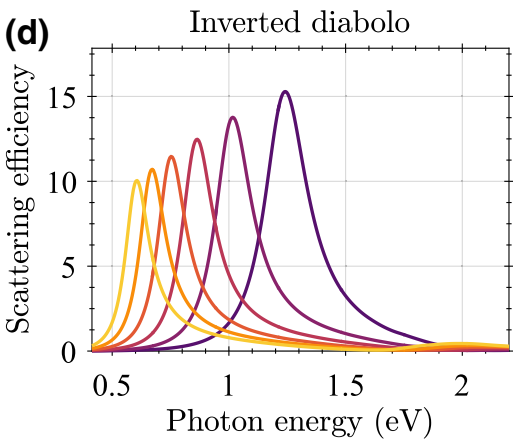

FIG. 2. Spectral dependence of the scattering efficiency $Q_{\text {scat }}$ of (a) bowtie, (b) diabolo, (c) inverted bowtie, (d) inverted diabolo PAs for several values of the wing length $v$ of the antennas.

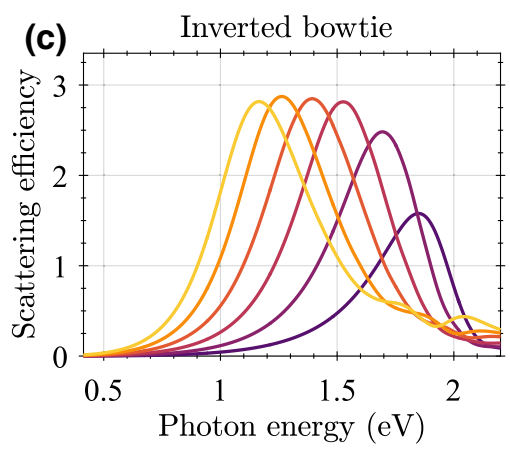

Wing length
$-50 \mathrm{~nm}$
$-70 \mathrm{~nm}$
$-90 \mathrm{~nm}$
$-110 \mathrm{~nm}$
$-130 \mathrm{~nm}$
$-150 \mathrm{~nm}$

Wing length

$-50 \mathrm{~nm}$

$-70 \mathrm{~nm}$

$-90 \mathrm{~nm}$

$-110 \mathrm{~nm}$

$-130 \mathrm{~nm}$

$-150 \mathrm{~nm}$ 

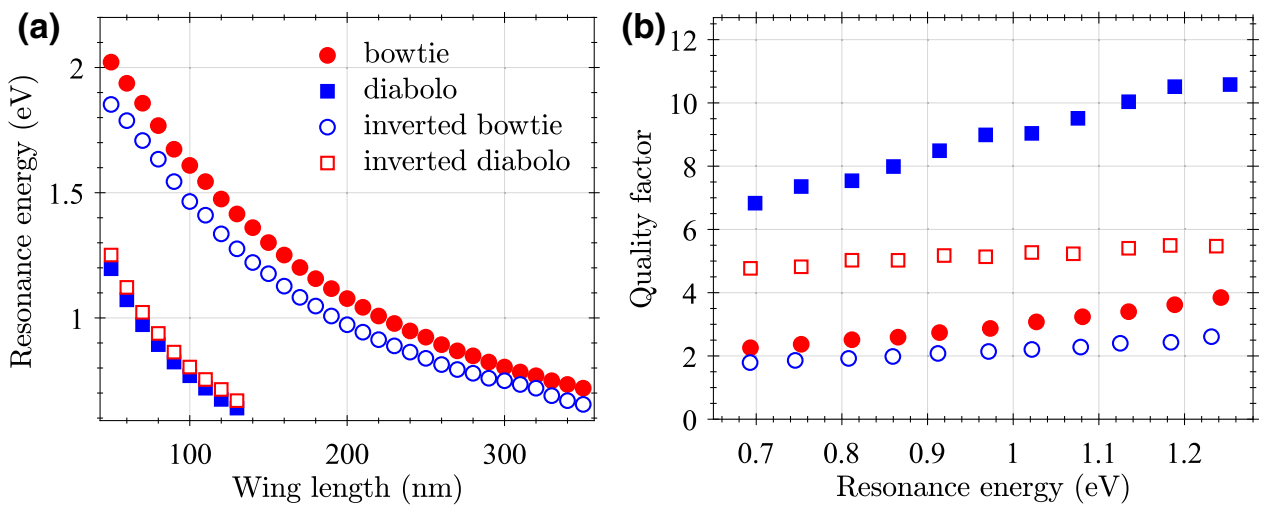

FIG. 3. (a) Peak energies of the scattering efficiency $Q_{\text {scat }}$ of bowtie (red full circles), diabolo (blue full squares), inverted bowtie (blue empty circles), and inverted diabolo (red empty squares) PAs as functions of the PA dimension-length of the wing $v$. (b) Quality factors of LSP (represented by the peak energy of scattering cross sections divided by their full width at half maximum). Notice that circles and squares correspond to bowtie and diabolo PAs, full and empty symbols correspond to particles (direct PAs) and apertures (inverted PAs), and red and blue color corresponds to electric and magnetic hot spots, respectively.

Not surprisingly, the peak energies of the scattering cross section for the diabolo PAs are considerably smaller than that for the bowtie PAs of the same wing length $v$. In other words, for the same energy, the diabolo PAs are smaller by a factor of more than 2 than the bowtie PAs. This effect is explained by a larger effective size of connected (i.e., diabolo) antennas in comparison with disjoint ones (bowtie), which can be seen from Figs. 1(a) and 1(b), and has been observed previously [44]. There is an important practical consequence. The bowtie geometry allows achievement of the high resonance energies for which diabolo-type PAs can be too small for an involved fabrication technique. Considering the minimum wing length of $50 \mathrm{~nm}$, diabolo antennas cover the LSP energy range up to $1.2 \mathrm{eV}$ while bowtie antennas operate up to $2.0 \mathrm{eV}$. On the other hand, diabolo geometry allows for a more compact PA design and better integration to more complex devices, such as a scanning near-field probe with the electric hot spot [45]. Further, their lower footprint represents an advantage for the fabrication of PA arrays, where a higher planar density of hot spots is achieved.

Diabolo antennas, either direct or inverted, feature considerably more intense and narrower scattering peaks corresponding to larger quality factors than bowtie antennas (Fig. 3). This is probably related to lower radiative losses due to their smaller volume.

In the following, we compare the properties and performance of all four types of PAs. We adjust the dimensions of the compared PAs so that they all feature the LSP resonance (LSPR) at the same energy. Table I shows the dimensions of the antennas for two specific energies: 1.8 $\mathrm{eV}$ corresponding to the minimum absorption of gold (i.e., minimum of the imaginary part of dielectric function) and $0.8 \mathrm{eV}$ corresponding to one of the optical communication wavelengths $(1550 \mathrm{~nm})$. We note that the former energy is accessible only with bowtie antennas. We therefore focus on the energy of $0.8 \mathrm{eV}$.
Figure 4 shows the formation of the hot spot. PAs featuring the lowest LSPR at the energy of $0.8 \mathrm{eV}$ are illuminated by a plane wave with the same photon energy. Bowtie and inverted diabolo PAs feature the electric hot spot and delocalized magnetic field, while diabolo and inverted bowtie PAs feature the magnetic hot spot and delocalized electric field. Interestingly, the volume of all the hot spots is comparable despite pronounced differences in the dimensions of PAs. The fields exhibit clear Babinet's complementarity: their spatial distribution in direct and complementary antennas is qualitatively similar with interchanged electric and magnetic components. Nevertheless, the magnitudes of the complementary fields differ rather significantly. As an example, the electric field within the hot spot of the bowtie antenna has the relative magnitude around 25 while the magnetic field of the inverted bowtie has the maximal relative magnitude less than 10 , i.e., almost three times weaker than expected. This observation is attributed to the finite thickness and conductivity of gold, which both limit the validity of Babinet's principle. As for the direct and inverted diabolo, the difference in the magnitudes of the electric and magnetic fields is less pronounced, but still quite significant. The bowtie and diabolo duality can be observed for the field forming the hot spot (e.g., electric for bowtie and magnetic for diabolo), which has a very similar spatial distribution in both cases. However, the distribution of the delocalized field (e.g., electric for bowtie and magnetic for diabolo) differs. In general, magnetic fields

TABLE I. Dimensions (wing length) of the antennas featuring the lowest LSPR at energies of 0.8 and $1.8 \mathrm{eV}$.

\begin{tabular}{ccccc}
\hline \hline & Bowtie & Diabolo & Inverted bowtie & Inverted diabolo \\
\hline $0.8 \mathrm{eV}$ & $300 \mathrm{~nm}$ & $95 \mathrm{~nm}$ & $270 \mathrm{~nm}$ & $100 \mathrm{~nm}$ \\
$1.8 \mathrm{eV}$ & $75 \mathrm{~nm}$ & $\ldots$ & $55 \mathrm{~nm}$ & $\ldots$ \\
\hline \hline
\end{tabular}


(a)

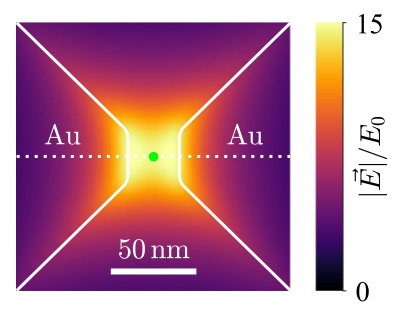

Bowtie
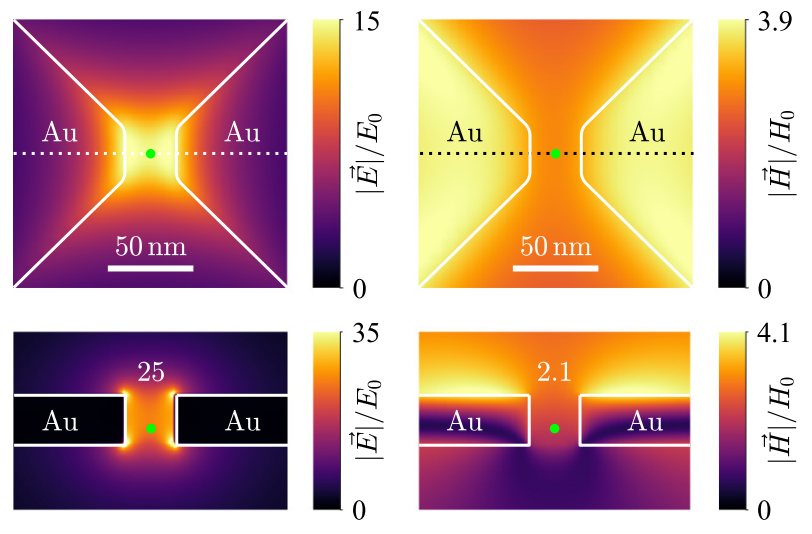

(c)

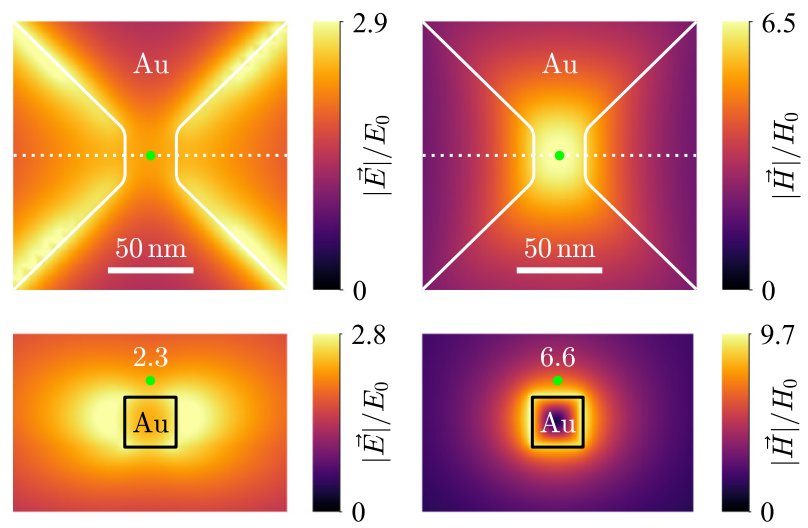

(b)

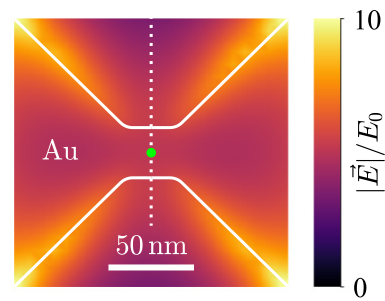

Diabolo
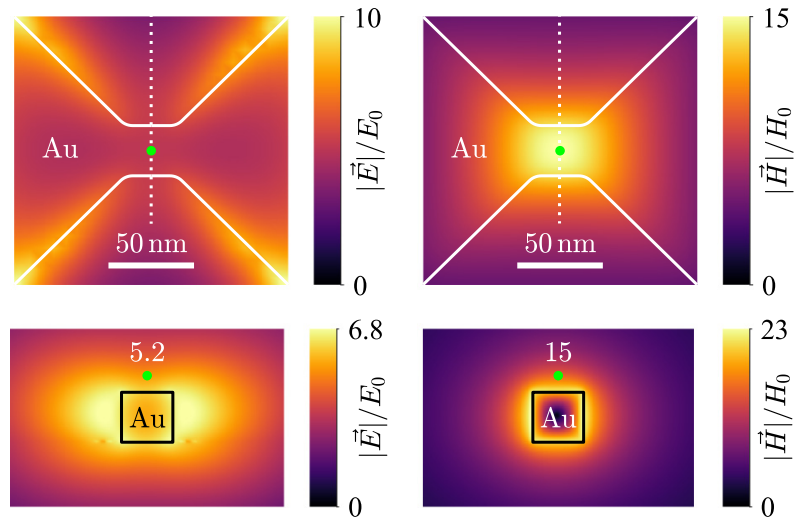

(d)

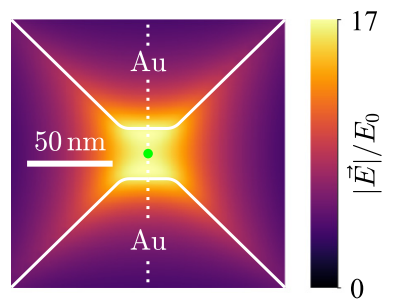

Inverted diabolo

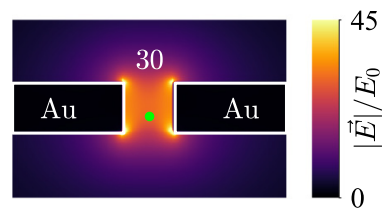

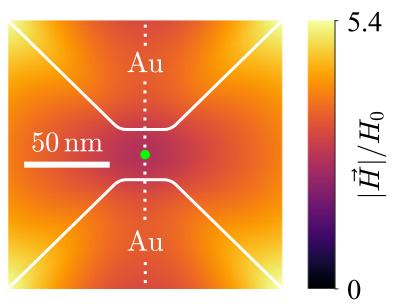

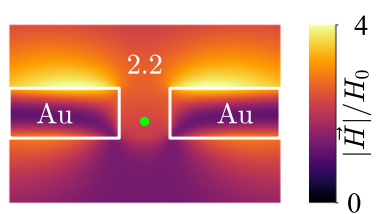

FIG. 4. Planar cross sections of the electric $(|\vec{E}|)$ and magnetic $(|\vec{H}|)$ field magnitudes divided by related magnitudes of the driving plane wave. The top two subplots in each panel show field distributions in the plane parallel to the PA plane, $10 \mathrm{~nm}$ above the upper PA boundary. The bottom two subplots then show field distributions in the vertical plane with the orientation indicated by white dotted lines in the in-plane field plots. The size of all PAs is set so that they feature the lowest LSPR at $0.8 \mathrm{eV}$, which is also the photon energy of the driving field. Solid white lines indicate antenna boundaries, while the hot spots are marked by the green point and the numbers correspond to the field enhancement in the hot spot. The figures show only the central part of the antennas with the metallic parts being denoted as Au for clarity.

are weaker than electric fields in agreement with previous findings $[42,46]$.

\section{B. Figures of merit for optical spectroscopy}

Plasmonic antennas can be used to enhance absorption and emission of light. Consequently, they enhance the signal of interest in various optical spectroscopy techniques, including absorption spectroscopy, Raman spectroscopy, photoluminescence spectroscopy, and absorption spectroscopy of magnetic transitions. Here we define figures of merit (FOM) for plasmonic enhancement of different spectroscopy techniques and evaluate them for all four types of PAs. We consider a small volume of the analyzed material (e.g., molecule, quantum dot, nanostructured material, or just nanosized crystal) that fits into the size of the hot spot.
In the case of absorption spectroscopy, absorbed power (per unit volume) can be expressed using Fermi's golden rule as $P=0.5 \operatorname{Re}[\sigma(\omega)]|E|^{2}$ where $\omega$ is the frequency of the probing radiation (in the following referred to as light), $\operatorname{Re}[\sigma]$ is the real part of the complex conductivity of the analyte, and $|E|$ is the magnitude of the electric component of light. For simplicity we consider that the transition dipole moment of the analyte is parallel to the electric field in the hot spot, which is polarized along the axis of the PA. The presence of plasmonic antennas alters the magnitude of electric field exciting the analyte. For the driving field (a plane wave) with the electric field intensity $E_{0}$, the electric intensity in the hot spot reads $E_{\mathrm{HS}}$. We define the electric field enhancement $Z_{E}=\left|E_{\mathrm{HS}}\right| / E_{0}$. Clearly, absorbed power is enhanced by the factor of $Z_{E}^{2}$, which is thus suitable FOM for plasmon-enhanced absorption spectroscopy. Raman scattering is a two-photon process, where each of the subprocesses, i.e., absorption of the driving 
photon and re-emission of the inelastically scattered photons, is enhanced by $Z_{E}^{2}$ (spectral dependence of $Z_{E}$ can be neglected considering a low relative energy shift in the Raman scattering and large energy width of plasmon resonances). Therefore, FOM for the plasmon-enhanced Raman spectroscopy reads $Z_{E}^{4}$.

Absorption spectroscopy of magnetic transitions is relevant for the study of rare-earth ions in the visible region $[20,21]$. Electron paramagnetic resonance is, in principle, also an absorption spectroscopy involving magnetic dipole transitions in the microwave spectral range. The absorbed power can be expressed as $P=0.5 \omega \operatorname{Im}[\mu(\omega)]|H|^{2}$ where $\omega$ is the frequency of light, $\operatorname{Im}[\mu]$ is the imaginary part of the complex permeability of the analyte, and $|H|$ is the magnitude of the magnetic component of light. For the magnetic field enhancement $Z_{H}$ defined analogously to $Z_{E}$, the FOM for absorption spectroscopy of magnetic transition reads $Z_{H}^{2}$.

We should note that the choice of the location in which we should evaluate the enhancement factors is somewhat arbitrary. In the case of the bowtie and inverted diabolo, we decide to take the values from a spot positioned in the center of the gap, $10 \mathrm{~nm}$ above the substrate, while for the inverted bowtie and diabolo, the spot is situated $10 \mathrm{~nm}$ above the center of the bridge. This choice gives us reasonable estimates that are close to the average values over the whole hot spots and it also ensures sufficient separation from the metal, which is relevant for dipolar emitters and their quenching. Ultimately, we can afford this slight inconsistency in the definition of the hot spot as we always compare a bowtie with an inverted diabolo and an inverted bowtie with a diabolo, i.e., PAs with the same definition of the hot spot. With this in mind, we can turn our attention back to the field enhancements $Z_{E}$ and $Z_{H}$. The inspection of Fig. 5 shows that both $Z_{E}$ and $Z_{H}$ decrease with increasing energy as a consequence of decreased funneling effect (the size of the wings decreases while the size of the bridge or the gap is kept constant). The electric field enhancement $Z_{E}$ ranges between 18 and 34 with the inverted diabolo PA having slightly better performance than the bowtie. The magnetic field-enhancement ranges between 10 and 17 for the diabolo PA but only between 4 and 8 for the inverted bowtie. Thus, the inverted diabolo presents an excellent option for the electric field enhancement while the inverted bowtie does not perform particularly well for the magnetic field enhancement.

Luminescence spectroscopy is another important method that can benefit from plasmon enhancement. We consider a simple model based on the rate equation. A metastable excitonic state with the degeneracy $g$ is populated through an external excitation with the rate $\gamma_{G}$. The generation is only efficient when the metastable state is unoccupied. For its population $n$, the total generation rate reads $(g-n) \gamma_{G}$. Excitons decay into the vacuum state via radiative and nonradiative recombination paths with the rates $\gamma_{R 0}$ and $\gamma_{\mathrm{NR} 0}$,

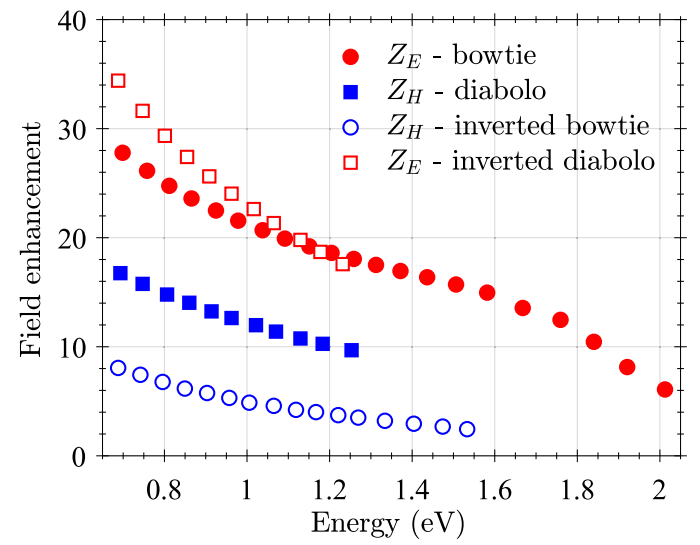

FIG. 5. Electric (red) and magnetic (blue) field enhancements for bowtie, diabolo, and their Babinet complements in their respective hot spots. The electric field enhancement $Z_{E}$, defined as the ratio of magnitudes of the local electric field $\left|\vec{E}_{\mathrm{HS}}\right|$ and the driving field $E_{0}$, enters the figures of merit for plasmon-enhanced absorption spectroscopy $\left(Z_{E}^{2}\right)$ and Raman scattering $\left(Z_{E}^{4}\right)$. On the other hand, the magnetic field enhancement $Z_{H}$, defined as the ratio of the magnitudes of the local magnetic field intensity $\left|\vec{H}_{\mathrm{HS}}\right|$ and the magnetic field intensity $H_{0}$ of the driving field, is important for plasmon-enhanced absorption spectroscopy of magnetic transitions $\left(Z_{H}^{2}\right)$.

respectively. The rate equation reads

$$
\frac{d n}{d t}=(g-n) \gamma_{G}-n \gamma_{R 0}-n \gamma_{\mathrm{NR} 0}
$$

In the steady state, $d n / d t=0$ and

$$
n=g \gamma_{G} /\left(\gamma_{G}+\gamma_{R 0}+\gamma_{\mathrm{NR} 0}\right) .
$$

Two regimes can be distinguished. In the linear (weak pumping) regime, $\gamma_{G} \ll \gamma_{R 0}+\gamma_{\mathrm{NR} 0}$ and

$$
n \approx g \gamma_{G} /\left(\gamma_{R 0}+\gamma_{\mathrm{NR} 0}\right),
$$

i.e., the population is proportional to pumping. In the saturation (strong pumping) regime, $\gamma_{G} \gg \gamma_{R 0}+\gamma_{\mathrm{NR} 0}$ and $n \approx$ $g$, i.e., the metastable state is fully occupied. The emitted power reads

$$
P_{\mathrm{PL}}=n \gamma_{R 0} \hbar \omega
$$

where $\hbar \omega$ is the photon energy. In the linear regime, the emitted power can be expressed using the internal quantum efficiency $\eta_{0}=\gamma_{R 0} /\left(\gamma_{R 0}+\gamma_{\mathrm{NR} 0}\right)$ as

$$
P_{l}=g \gamma_{G} \eta_{0} \hbar \omega
$$

and in the saturation regime

$$
P_{s}=g \gamma_{R 0} \hbar \omega .
$$

The presence of plasmonic antennas affects all three processes (generation, radiative decay, and nonradiative 
decay). The effect on generation varies from very important in the case of photoluminescence [18] to negligible in the case of electroluminescence. In general, generation is a sequential inelastic process and cannot be described by a simple model. For that, we do not consider plasmon enhancement of generation in the following and focus on its influence on the radiative and nonradiative decay rates.

Spontaneous emission is affected via Purcell effect [47]. The emitter transfers its energy to PA where it is partially radiated into far field and partially dissipated. It is customary to express the rates of both processes in multiples of the spontaneous emission rate $\gamma_{R 0}: Z_{R}$ being the radiative enhancement and $Z_{\mathrm{NR}}$ the nonradiative enhancement $[48,49]$. The total radiative and nonradiative decay rates in the presence of plasmonic particles read $\gamma_{R}=Z_{R} \gamma_{R 0}$ and $\gamma_{\mathrm{NR}}=Z_{\mathrm{NR}} \gamma_{R 0}+\gamma_{\mathrm{NR} 0}$, respectively, and the internal quantum efficiency reads $\eta=\gamma_{R} /\left(\gamma_{R}+\gamma_{\mathrm{NR}}\right)$.

The figure of merit for plasmon-enhanced luminescence (only its emission part) is the rate of the powers emitted with and without the presence of the PA. For the linear regime, FOM is

$$
F_{l}=\eta / \eta_{0},
$$

while for the saturation regime it reads simply

$$
F_{s}=Z_{R} .
$$

Consequently, only emitters with a poor internal quantum efficiency can benefit from plasmon enhancement in the linear regime while the emitters with a high internal quantum efficiency will suffer from the dissipation in metallic
PA. On the other hand, in the saturation regime plasmon enhancement is benefitable as long as $Z_{R}>1$.

Figure 6 shows the spectral dependence of radiative and nonradiative enhancement factors $\left(Z_{R}\right.$ and $Z_{\mathrm{NR}}$, respectively) for different types of PA with the maximum field enhancement at $0.8 \mathrm{eV}$. A pointlike isotropic emitter (i.e., all polarizations are involved with the same intensity) is positioned in the center of the PA $10 \mathrm{~nm}$ above the substrate (bowtie and inverted diabolo) or $10 \mathrm{~nm}$ above the surface of gold (diabolo and inverted bowtie). Such a separation shall suppress emission quenching due to nonradiative decay of the emitter. For the electric dipole transitions, a large radiative enhancement (several hundreds) is obtained for both the bowtie and inverted diabolo. The inverted diabolo offers approximately twice larger peak enhancement [Fig. 6(a)] than the bowtie PA. However, the bowtie benefits from a much lower nonradiative enhancement and is thus preferable for most emitters in the linear regime [Fig. 6(c)]. For magnetic dipole transitions, the diabolo provides a considerably larger radiative enhancement than the inverted bowtie, but it also suffers from the considerably larger nonradiative enhancement [Fig. 6(b)]. In addition, the resonance of the inverted bowtie is considerably wider, which can prioritize this type of antenna for emitters with broad spectral bands. The preferred PA type therefore depends on specific application. We note that the peaks in the enhancement are spectrally shifted from the maximum field enhancement; the effect is particularly pronounced for the inverted bowtie.

At this point it is worthwhile to estimate the accuracy of the electromagnetic simulations and the relevance
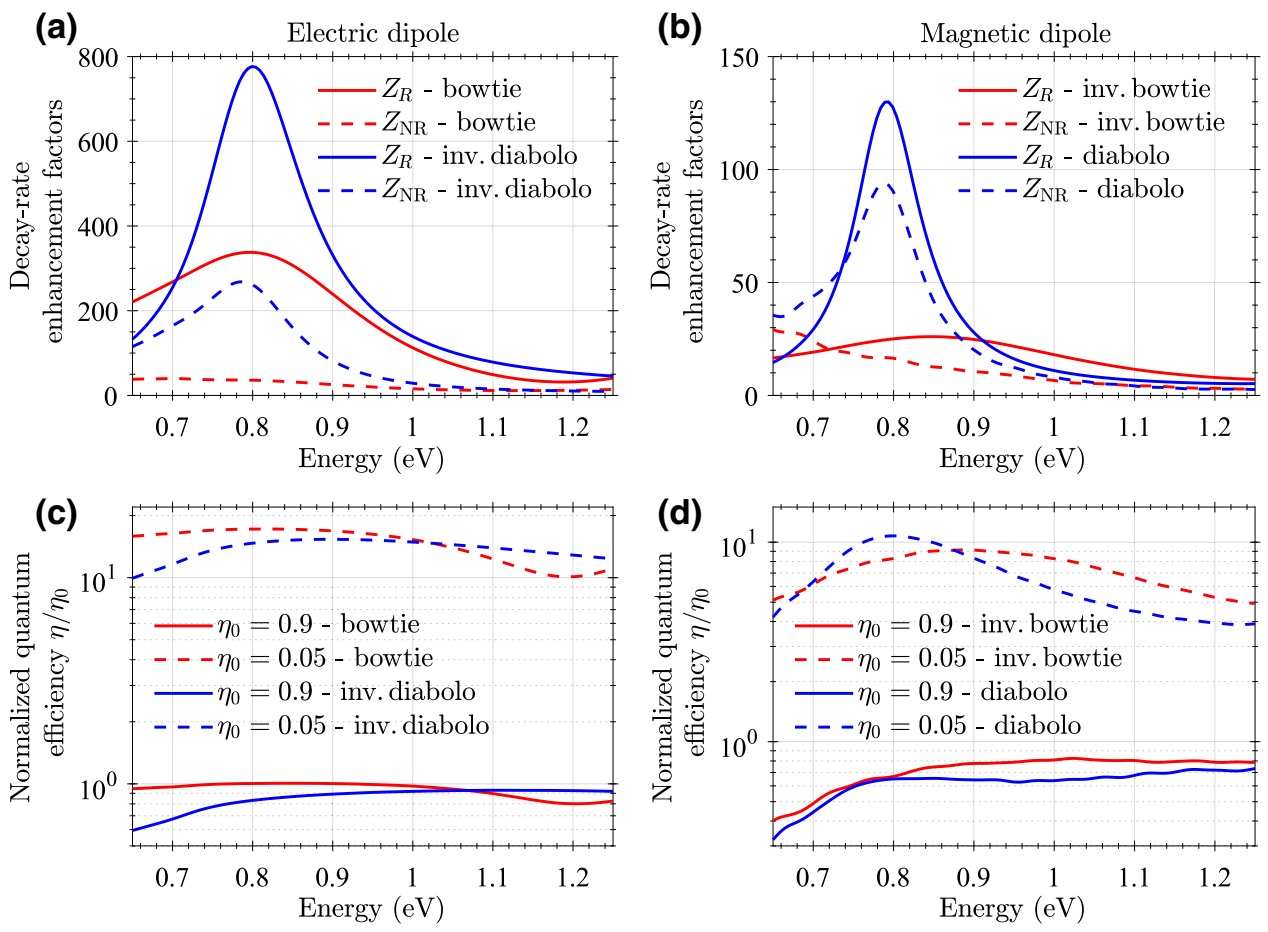

FIG. 6. Radiative and nonradiative enhancement factors $Z_{R}$ and $Z_{\mathrm{NR}}$, respectively, as functions of the photon energy for the (a) electric dipole transition and (b) magnetic dipole transition. The values are averaged over all possible polarizations of the transitions. Panels (c) and (d) then show enhancement of the overall quantum efficiency for two values of the internal quantum efficiency, namely $\eta_{0}=$ 0.9 (good emitter) and $\eta_{0}=0.05$ (poor emitter). Note that even though the radiative enhancement for direct and inverted diabolos is significantly higher than for their bowtie counterparts, their enhancement of quantum efficiency is due to their equally larger nonradiative enhancement more or less the same. 
of their results for the experiment. Several authors compared experimental and calculated values of luminescence enhancement factors in various systems, including organic dyes coupled to bowtie antennas [18] (measured, up to 1340; calculated, 1690), $\mathrm{MoS}_{2}$ coupled to bowtie antennas [50] (measured, 980; calculated, 982), and $\mathrm{WSe}_{2}$ monolayer coupled to cavity-backed slot antennas [51] (measured: 340; calculated: 400). Apparently, there is a reasonable agreement between simulations and experiment with differences up to $20 \%$. Further, the differences consist in a rather systematic overestimation of calculations due to model simplifications. Hence, the conclusions based on the simulations shall be robust and reproducible in the experiment.

\section{Optical trapping}

Apart from enhancing optical processes, electric hot spots can also serve as optical tweezers, i.e., they can be used to trap small objects. In the dipole limit, the optical force that facilitates this trapping is proportional to the gradient of the square of the electric field. Considering the evanescent nature of plasmonic near fields, electric hot spots in the vicinity of plasmonic structures can lead to very strong forces, stronger even than those encountered in tightly focused beams that are routinely used to manipulate both metallic and dielectric particles, living cells, DNA, or bacteria [52].

To estimate the strength of the trapping force provided by our structures, we calculated the energy required by a $20-\mathrm{nm}$ polystyrene sphere to escape the potential well formed by the plasmonic hot spot. Considering the small size of the particle, we deem it sufficient to work within the dipole approximation, where the potential energy of the small sphere reads [53]

$$
V=-\frac{\alpha}{2}|\vec{E}|^{2},
$$

where $\alpha$ is the polarizability of the polystyrene particle (permittivity 2.46). Note that we limit ourselves only to structures with electric hot spots as the magnetic dipole moment and consequently also the magnetic trapping potential for such a small polystyrene particle is negligible. Considering an illumination in the form of a $1-\mathrm{mW}$ beam focused into a $2-\mu \mathrm{m}$ spot (corresponding to the intensity of $318 \mathrm{kWm}^{-2}$ ) with the investigated structure in its centre, the magnitudes of the trapping potential (which we define as the potential difference between the hot spot and when we are away from it) are plotted in Fig. 7. As one might expect, the trapping potential mimics the field enhancement shown in Fig. 5, with larger values attained at lower resonance energies. Assuming that the system is at the room temperature $(T=300 \mathrm{~K})$, the trapping energy at $0.8 \mathrm{eV}$ becomes comparable to the thermal energy of the polystyrene particles, which indicates that these structures

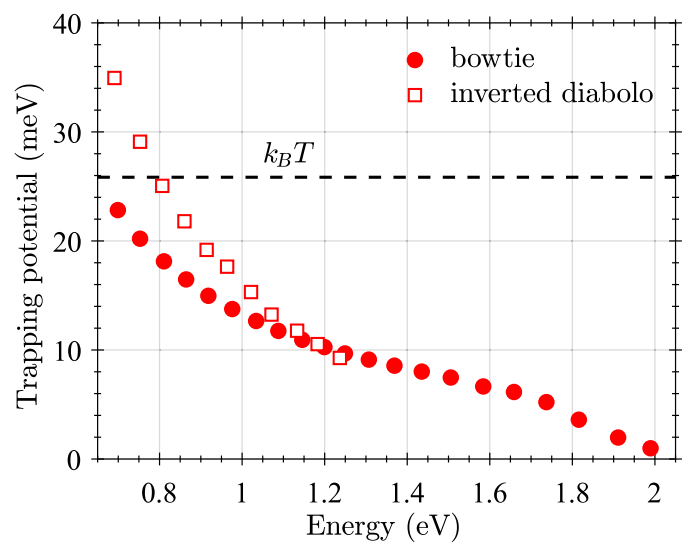

FIG. 7. Depth of the trapping potential for $20-\mathrm{nm}$ polystyrene spheres induced by bowtie and inverted diabolo PAs illuminated by the light with the intensity of $318 \mathrm{kWm}^{-2}$. The potential is expressed at the energy of the lowest LSPR and displayed as a function of this energy. The dashed line indicates the thermal energy at room temperature $\left(k_{B} T=26 \mathrm{meV}\right)$.

have a potential to serve as a dual purpose tool that is capable of capturing an object and enhance its optical response at nanoscale.

\section{Materials beyond gold}

To demonstrate the general validity of our results we now discuss two other plasmonic materials: silver and zirconium nitride $(\mathrm{ZrN})$. Silver is a traditional plasmonic metal, which supports surface plasmon polaritons (SPP) in a broader spectral range than gold (up to the energy of $3 \mathrm{eV}$ ) and exhibits slightly lower electron scattering rate. On the other hand, silver PAs are not as stable as gold PAs and can degrade over time [54]. ZrN is a semiconductor from a class of transition-metal nitrides that supports SPP in the visible spectral range (up to $3 \mathrm{eV}$ ) under sufficiently large doping [55]. The electron scattering in $\mathrm{ZrN}$ is significantly larger than in gold. For our simulations, the dielectric function of silver has been taken from Johnson and Christy [43] and the dielectric function of the doped $\mathrm{ZrN}$ has been taken from Naik et al. [55]. The electron scattering time $\tau$ can be estimated from the dielectric function $\epsilon(\omega)$ as $\tau=-\operatorname{Im}(\epsilon) /[\omega \operatorname{Re}(\epsilon)]$, where Re and $\operatorname{Im}$ denote the real and imaginary part, and $\omega$ is the angular frequency. At the photon energy of $0.8 \mathrm{eV}, \tau$ reads $32 \mathrm{fs}$ for silver, 8.2 fs for gold, and 0.8 fs for $\mathrm{ZrN}$.

The energies of the lowest LSPR supported by silver and ZrN PAs are shown as Figs. S1(a) and S2(a) within the Supplemental Material [56]. The energy is only weakly dependent on the material of the PA, with a slight blueshift observed for the smallest silver PAs attributed to the large plasma frequency and the absence of interband transitions in silver in the respective energy range. The energies of LSPR in the Babinet-complementary PAs are nearly identical, with the differences up to $10 \%$ for gold and silver. 
Notably, $\mathrm{ZrN}$ exhibits larger differences (up to $15 \%$ ) at the energy of $2 \mathrm{eV}$ (due to proximity of the plasma frequency and a related zero-cross-point of the real part of the dielectric function) but very low differences (below 5\%) at energies below $1 \mathrm{eV}$ where $\mathrm{ZrN}$ rapidly approaches the limit of perfect electric conductor.

The $Q$ factors of the lowest LSPR supported by silver and ZrN PAs are shown as Figs. S1 and S2 within the Supplemental Material [56]. Typical values for silver PAs are by about $25 \%$ higher than for gold PAs, while the values for $\mathrm{ZrN}$ PAs are about four times lower. As expected, the $Q$ factor decreases for decreasing electron scattering time. The relation is weaker than linear, since the total plasmon damping is in addition to the collision damping contributed also by the radiation damping and the Landau damping [57].

Figure 8 compares all three materials in terms of the figures of merit of optical spectroscopy and trapping. We show the results only for the better PA of the pair supporting a specific hot spot, which is the inverted diabolo PA for the electric hot spot and the diabolo PA for the magnetic hot spot. Complete characterization including also the bowtie and inverted bowtie PAs is provided as Figs. S1 and S2 within the Supplemental Material [56]. The trends observed for gold PAs are qualitatively reproduced for silver and $\mathrm{ZrN}$ PAs. Low-scattering materials provide better FOM. The effect is most pronounced for the decay-rate enhancement factors, where the low scattering results in both high radiative decay-rate enhancement $Z_{R}$ and low nonradiative decay-rate enhancement $Z_{\mathrm{NR}}$.

Tables II and III summarize the FOM at the energy of $0.8 \mathrm{eV}$ corresponding to the telecommunication wavelength of $1.55 \mu \mathrm{m}$. The dimensions of the antennas are adjusted to set the energy of the lowest LSPR to $0.8 \mathrm{eV}$ (represented by the peak of the scattering cross section) so that we evaluate the resonant (maximum) values of the FOM. The antenna footprint $F$ is defined as the area of smallest rectangle fully containing the antenna. Table II presents the FOM related to the electric hot spot: the $Q$ factor defined as the peak energy divided by the FWHM of the scattering cross section, the electric field enhancement $Z_{E}$, the radiative $\left(Z_{R}\right)$ and nonradiative $\left(Z_{\mathrm{NR}}\right)$ decay-rate
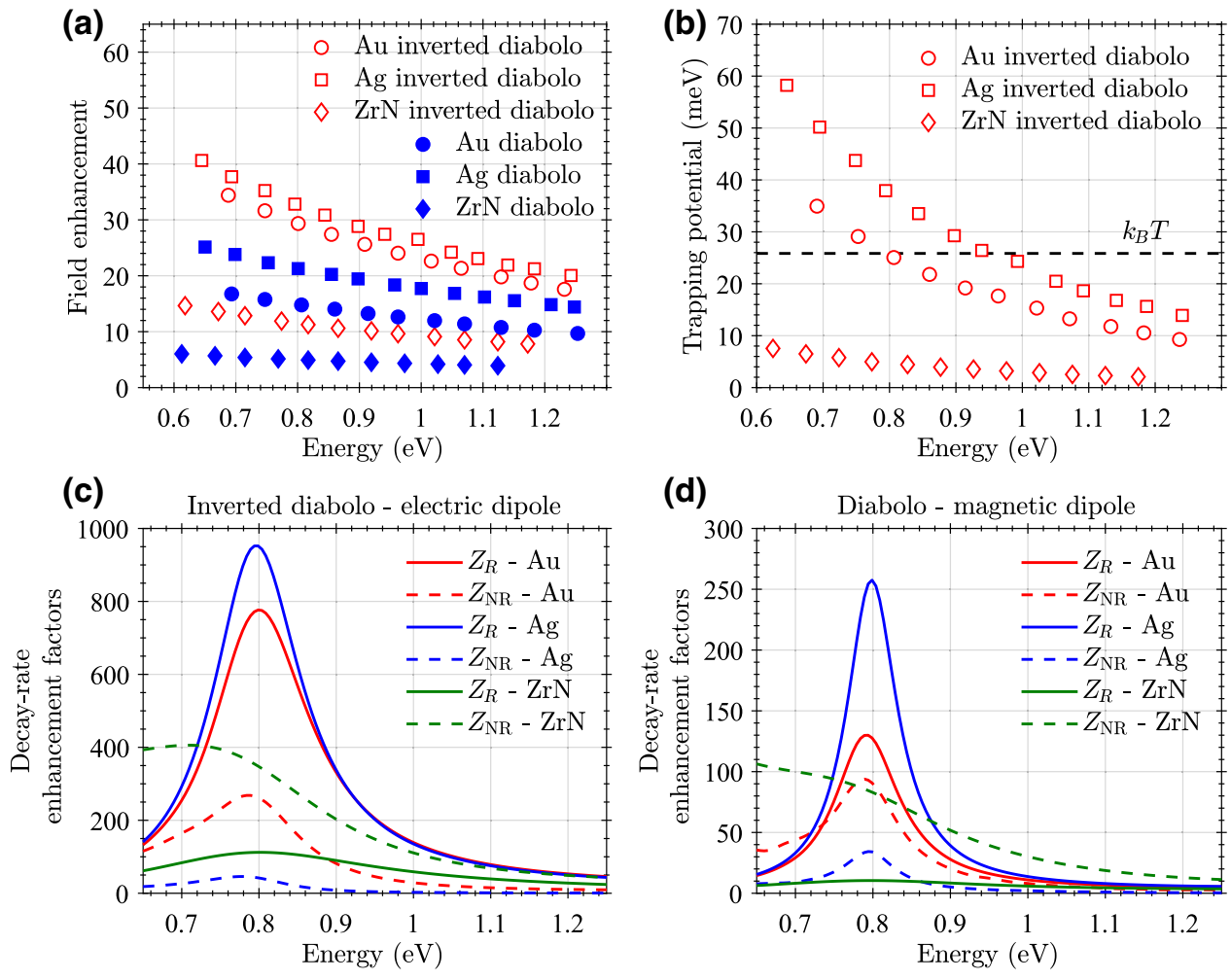

FIG. 8. Comparison of gold, silver, and ZrN PAs. (a) Field-enhancement factors for the electric field in inverted diabolo PAs (red) and the magnetic field in diabolo PAs (blue) expressed at the energy of the lowest LSPR as functions of this energy. (b) Depth of the trapping potential for 20-nm polystyrene spheres induced by inverted diabolo PAs illuminated by the light with the intensity of $318 \mathrm{kWm}^{-2}$. The potential is expressed at the energy of the lowest LSPR and displayed as a function of this energy. The dashed line indicates the thermal energy at room temperature $\left(k_{B} T=26 \mathrm{meV}\right)$. (c),(d) Radiative and nonradiative enhancement factors $Z_{R}$ and $Z_{\mathrm{NR}}$, respectively, as functions of the photon energy for (c) the inverted diabolo PA and the electric dipole transition and (d) the diabolo PA and the magnetic dipole transition. The values are averaged over all possible polarizations of the transitions. The energy of the lowest LSPR in the PAs is set to $0.8 \mathrm{eV}$. 
TABLE II. Figures of merit related to the electric hot spot at the energy of $0.8 \mathrm{eV}$.

\begin{tabular}{|c|c|c|c|c|c|c|c|}
\hline Material & Geometry & $F\left[\mathrm{~nm}^{2}\right]$ & $Q$ & $Z_{E}$ & $Z_{R}$ & $Z_{\mathrm{NR}}$ & $V_{\mathrm{TB}}[\mathrm{meV}]$ \\
\hline \multirow[t]{2}{*}{ Silver } & i. diabolo & $5.8 \times 10^{4}$ & 5.6 & 33 & 952 & 48 & 37 \\
\hline & bowtie & $41 \times 10^{4}$ & 2.4 & 24 & 304 & 1 & 16 \\
\hline \multirow[t]{2}{*}{ Gold } & i. diabolo & $5.3 \times 10^{4}$ & 4.9 & 30 & 775 & 268 & 25.6 \\
\hline & bowtie & $40 \times 10^{4}$ & 2.4 & 25 & 338 & 37 & 19.4 \\
\hline \multirow[t]{2}{*}{$\mathrm{ZrN}$} & i. diabolo & $5.3 \times 10^{4}$ & 2.1 & 12 & 112 & 350 & 5.7 \\
\hline & bowtie & $36 \times 10^{4}$ & 1.6 & 16 & 142 & 140 & 7.3 \\
\hline
\end{tabular}

enhancements for an electric dipole transition, and the trapping barrier $V_{\mathrm{TB}}$ defined as the depth of the trapping potential for the illumination intensity of $318 \mathrm{kWm}^{-2}$. It includes only PAs with the electric hot spot, i.e., bowtie and inverted diabolo. Table III presents the FOM related to the magnetic hot spot: the $Q$ factor, the magnetic field enhancement $Z_{H}$, and the radiative $\left(Z_{R}\right)$ and nonradiative $\left(Z_{\mathrm{NR}}\right)$ decay-rate enhancements for a magnetic dipole transition. It includes PAs with the magnetic hot spot, i.e., inverted bowtie and diabolo.

There is a clear qualitative correlation between all FOM. Large values of $Q$ factor correspond to a large field enhancement and large radiative decay enhancements, while nonradiative decay enhancements are small. Silver with the highest electron scattering time of all three materials involved in the study shows better performance compared to gold, which is superior to $\mathrm{ZrN}$, the material with the lowest electron scattering time. The diabolo PAs offer better FOM (and lower footprint) than the bowtie PAs due to reduced radiation losses. This finding holds for both the bowtie and the inverted diabolo PAs supporting the electric hot spot and the inverted bowtie and the diabolo PAs supporting the magnetic hot spot.

\section{E. Babinet dimer with electromagnetic hot spot}

Bowtie and diabolo PAs enhance either an electric or a magnetic component of the field, while the other component is only weakly enhanced and spatially focused. In this section we propose a Babinet dimer antenna that forms an electromagnetic hot spot enhancing and focusing both components of the electromagnetic field equally. The Babinet dimer antenna is formed by a direct and an inverse PA, vertically stacked so closely that their individual electric and magnetic hot spots overlap. We explore and compare two configurations, namely the Babinet bowtie dimer (BBD) [schematically depicted in Fig. 9(a)], consisting of a bowtie on top of an inverted bowtie PA, and the Babinet diabolo dimer (BDD) [sketched in Fig. 9(c)] made up by an inverted diabolo on top of a diabolo PA. In both configurations, the upper PA is rotated with respect to the bottom one by $90^{\circ}$ so that both of them can be excited by the same source polarization (oriented along the long axis of the direct PA) and the upper and bottom PAs are separated by a $10-\mathrm{nm}$ spacer layer with the refractive index equal to 1.5. As the individual modes in the closely spaced PAs exhibit strong interaction, the dimensions of the dimer constituents are adjusted so that the maximum field enhancement occurs at $0.8 \mathrm{eV}$ for both the electric and magnetic component. For the BBD, the wing lengths of the top $(\uparrow)$ and bottom $(\downarrow)$ PAs are set to $v_{\uparrow}=110 \mathrm{~nm}$ and $v_{\downarrow}=200 \mathrm{~nm}$, while for the BDD, the optimal dimensions $\operatorname{read} v_{\uparrow}=200 \mathrm{~nm}$ and $v_{\downarrow}=110 \mathrm{~nm}$. Note that the antenna providing the magnetic enhancement is in both cases situated underneath the one with the electric enhancement so that the electromagnetic hot spot is directly accessible from the top.

Figure 9(b) demonstrates the formation of the electromagnetic hot spot in the BBD. The dimer is illuminated by the field polarized along the long axis of the bowtie and perpendicular to the long axis of inverted bowtie, which results in formation of an electric hot spot (field enhancement 14) around the direct bowtie and a magnetic hot spot (field enhancement 13) around the inverted bowtie. The closely spaced hot spots overlap, yielding the maximum simultaneous enhancement of both fields close to 8.4 at the position indicated by the green point in Fig. 9. The inspection of Figs. 3 and 5 reveals that these values are similar

TABLE III. Figures of merit related to the magnetic hot spot at the energy of $0.8 \mathrm{eV}$.

\begin{tabular}{|c|c|c|c|c|c|c|}
\hline Material & Geometry & $F\left[\mathrm{~nm}^{2}\right]$ & $Q$ & $Z_{H}$ & $Z_{R}$ & $Z_{\mathrm{NR}}$ \\
\hline \multirow[t]{2}{*}{ Silver } & diabolo & $4.8 \times 10^{4}$ & 9.7 & 21 & 257 & 34 \\
\hline & i. bowtie & $34 \times 10^{4}$ & 1.9 & 7.5 & 40 & 5 \\
\hline \multirow[t]{2}{*}{ Gold } & diabolo & $4.8 \times 10^{4}$ & 7.5 & 15 & 130 & 94 \\
\hline & i. bowtie & $33 \times 10^{4}$ & 1.9 & 6.6 & 26 & 15 \\
\hline \multirow[t]{2}{*}{$\mathrm{ZrN}$} & diabolo & $4.4 \times 10^{4}$ & 2.1 & 5.0 & 11 & 83 \\
\hline & i. bowtie & $31 \times 10^{4}$ & 1.0 & 3.7 & 8 & 55 \\
\hline
\end{tabular}




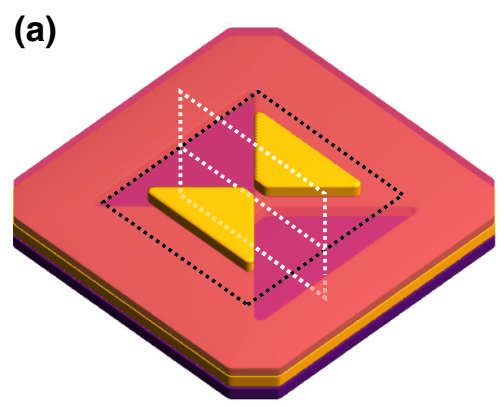

(b)

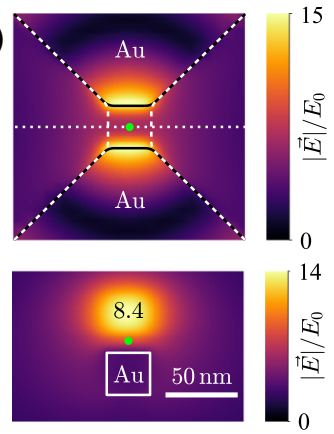

(c)

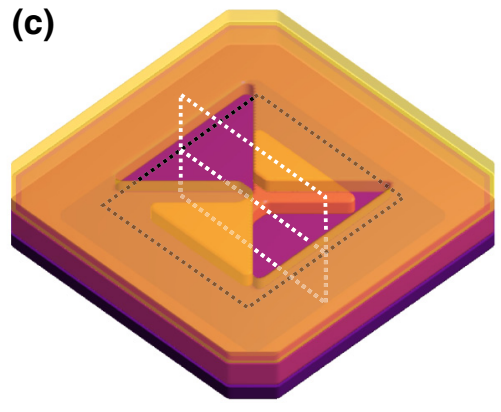

(d)
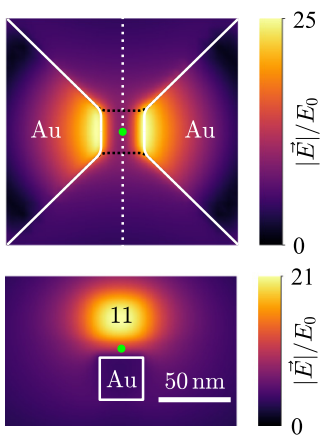
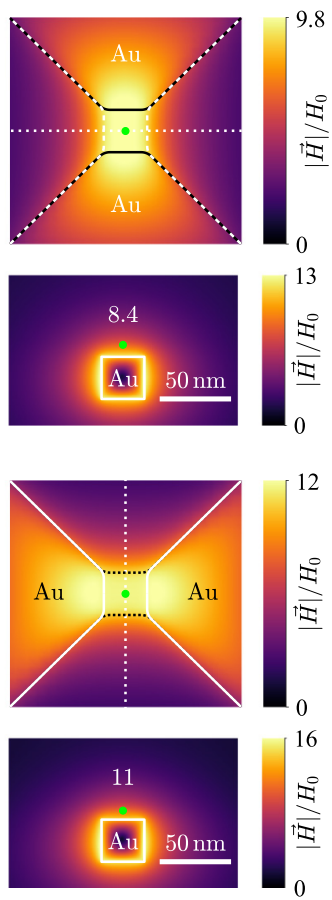

FIG. 9. (a) Schematic drawing of the BBD. A direct bowtie (wing length $110 \mathrm{~nm}$ ) lies on top of an inverted bowtie (wing length $200 \mathrm{~nm}$ ), they are mutually rotated by $90^{\circ}$ and separated by a 10-nm spacer layer. (b) Distribution of the electric (left) and magnetic (right) fields in the vicinity of the BBD. The top two subplots show the fields in a plane parallel to the individual PAs, namely inside the spacer layer with 5-nm distance from both of them [indicated by a black dotted line in (a)]. The bottom two subplots then show the fields in a vertical plane perpendicular to the metal bridge of the inverted bowtie. The orientation of the plane is outlined by a white dotted line in the schematic drawing in (a) and also in the top two subplots showing the fields in the horizontal plane. (c) Schematic drawing of the BDD. A direct diabolo (wing length $110 \mathrm{~nm}$ ) lies underneath an inverted bowtie (wing length $200 \mathrm{~nm}$ ), they are mutually rotated by $90^{\circ}$ and separated by a $10-\mathrm{nm}$ spacer layer. The distribution of the electric (left) and the magnetic (right) fields around the BDD is plotted in (d), with planar cross sections positioned and oriented in the same manner as in the case of BBD. Note that both BBD and BDD are illuminated with a plane wave polarized along the long axes of the direct PAs and the green point marks the position, in which the electric and magnetic field enhancements are equal in magnitude (with the value specified by the number).

to those obtained for single PAs of comparable size. This indicates that the enhancement mechanism based on the charge accumulation (or the current funneling) at the wing apices is rather robust and resistant to changes in surroundings of the PA. The other proposed design, BDD, possesses electromagnetic hot spot as well, with maximum simultaneous enhancement of 11, while the individual maxima read 21 (electric enhancement) and 16 (magnetic enhancement) [see Fig. 9(d)]. These values are again close to those encountered in single PAs, despite the partial screening of the bottom diabolo by its upper counterpart. On the whole, the better performance of isolated diabolos (at least in terms of local field enhancement) imprints itself also into Babinet dimers.

So far we altogether disregard the vectorial nature of electromagnetic fields, which can be important in certain applications. In the designs proposed above, the electric and magnetic fields in the hot spot are perpendicular to each other, but one can achieve also other mutual orientations simply by rotating the vertically stacked antennas with respect to each other. Such control over the local polarization state of the light is quite valuable, especially when we consider the aforementioned robustness with both the field amplitude and orientation tightly bound to the geometry of the PAs.

In comparison to previous proposals $[58,59]$ and realizations [13] of plasmonic electromagnetic hot spots, our proposal brings two benefits. (i) It enhances both fields on equal basis, i.e., with the same amplitude, resonance frequency, and lateral spatial distribution. (ii) It involves two isolated antennas, which can be adjusted independently, allowing extended tunability of the hot spot.

Interestingly, BBD can be used as Huygens metaatoms - building blocks of metasurfaces that simultaneously support electric and magnetic modes. Due to the so-called Kerker effect [60] the Huygens metasurfaces exhibit strongly directional scattering of light and allow achievement of metasurfaces with improved transmission efficiency [61]. The realization of Huygens meta-atom reported by Zhang et al. [61] is composed of a direct rodlike plasmonic antenna and an inverted rodlike plasmonic aperture positioned above each other, i.e., it can be 
viewed as a BBD with bowties replaced with rods. Using bowtie antennas instead of rods shall preserve the directional scattering and enhance the light-matter coupling in the hot spot, paving the way towards multifunctional metasurfaces capable of simultaneous enhancement the light-matter interaction and light manipulation with high transmission efficiency. We note that the BBD discussed above is optimized for the field enhancement and exhibits rather low directionality of the scattering. Simultaneous optimization of both quantities is a subject for further investigation.

Ref. [61] also provides a possible fabrication protocol for the BBD. Alternatively, electron-beam lithography overlay process can be used $[62,63]$.

\section{CONCLUSION}

We perform a comprehensive study on the plasmonic antennas featuring electric, magnetic, and electromagnetic hot spots: bowtie and inverted diabolo, diabolo and inverted bowtie, and their dimers, respectively. We combine two types of electric-magnetic complementarity: bowtie and diabolo duality and Babinet's principle.

For a specific resonance frequency, diabolo antennas are significantly smaller than bowtie antennas, and thus harder to fabricate but easier to integrate. For the minimum wing length of $50 \mathrm{~nm}$, bowties covered the energy range up to $2.0 \mathrm{eV}$ while diabolos only up to $1.2 \mathrm{eV}$. Diabolo antennas also exhibit considerably narrower resonances related to a higher $Q$ factor as a consequence of a lower scattering cross section.

We evaluate figures of merit for different methods of optical spectroscopy and for optical trapping. One of the most important figures of merit is the field enhancement in the hot spot, which is larger for the diabolo antennas than for the bowtie antennas (and also for the electric field than the magnetic field). For the luminescence, the key figure of merit is the radiative and nonradiative decay enhancement. Here, diabolo antennas exhibited a slightly stronger radiative decay enhancement than bowtie antennas but also pronouncedly stronger nonradiative enhancement, making the direct bowtie antenna a preferred option for the electric dipole transitions and the inverted bowties an equivalent alternative of the diabolo for the magnetic dipole transitions.

As for the material composition, we find better figures of merit for the plasmonic antennas made of materials with lower electron scattering rates. Silver is slightly better than gold, while zirconium nitride is considerably worse.

Finally, we propose Babinet dimer antennas enhancing both the electric and magnetic field on an equal basis and forming an electromagnetic hot spot, which finds applications in studies of rare earth ions, optical trapping, metamaterials, or nonlinear optics.

\section{ACKNOWLEDGMENTS}

We acknowledge the support by the Czech Science Foundation (Grant No. 17-25799S) and Ministry of Education, Youth and Sports of the Czech Republic (Project CEITEC 2020, No. LQ1601). Part of the work is supported by European Union's Horizon 2020 (Project PETER, No. 767227).

[1] L. Novotny and N. van Hulst, Antennas for light, Nat. Photonics 5, 83 (2011).

[2] S. Nie and S. R. Emory, Probing single molecules and single nanoparticles by surface-enhanced Raman scattering, Science 275, 1102 (1997).

[3] J. R. Krenn, A. Dereux, J. C. Weeber, E. Bourillot, Y. Lacroute, J. P. Goudonnet, G. Schider, W. Gotschy, A. Leitner, F. R. Aussenegg, and C. Girard, Squeezing the Optical Near-Field Zone by Plasmon Coupling of Metallic Nanoparticles, Phys. Rev. Lett. 82, 2590 (1999).

[4] J.-C. Weeber, J. R. Krenn, A. Dereux, B. Lamprecht, Y. Lacroute, and J. P. Goudonnet, Near-field observation of surface plasmon polariton propagation on thin metal stripes, Phys. Rev. B 64, 045411 (2001).

[5] H. Gao, Q. Cao, M. Zhu, D. Teng, and S. Shen, Nanofocusing of terahertz wave in a tapered hyperbolic metal waveguide, Opt. Express 22, 32071 (2014).

[6] M. Urbieta, M. Barbry, Y. Zhang, P. Koval, D. SánchezPortal, N. Zabala, and J. Aizpurua, Atomic-scale lightning rod effect in plasmonic picocavities: A classical view to a quantum effect, ACS Nano 12, 585 (2018).

[7] F. Benz, M. K. Schmidt, A. Dreismann, R. Chikkaraddy, Y. Zhang, A. Demetriadou, C. Carnegie, H. Ohadi, B. de Nijs, R. Esteban, J. Aizpurua, and J. J. Baumberg, Singlemolecule optomechanics in "picocavities", Science 354, 726 (2016).

[8] L. Zhou, Q. Gan, F. J. Bartoli, and V. Dierolf, Direct nearfield optical imaging of UV bowtie nanoantennas, Opt. Express 17, 20301 (2009).

[9] A. Lee, G. F. S. Andrade, A. Ahmed, M. L. Souza, N. Coombs, E. Tumarkin, K. Liu, R. Gordon, A. G. Brolo, and E. Kumacheva, Probing dynamic generation of hotspots in self-assembled chains of gold nanorods by surfaceenhanced Raman scattering, J. Am. Chem. Soc. 133, 7563 (2011).

[10] T. Grosjean, M. Mivelle, F. I. Baida, G. W. Burr, and U. C. Fischer, Diabolo nanoantenna for enhancing and confining the magnetic optical field, Nano Lett. 11, 1009 (2011).

[11] M. Lorente-Crespo, L. Wang, R. Ortuno, C. García-Meca, Y. Ekinci, and A. Martínez, Magnetic hot spots in closely spaced thick gold nanorings, Nano Lett. 13, 2654 (2013).

[12] I. C. Bicket, E. P. Bellido, S. Meuret, A. Polman, and G. A. Botton, Correlative electron energy loss spectroscopy and cathodoluminescence spectroscopy on three-dimensional plasmonic split ring resonators, Microscopy 67, i40 (2018).

[13] Y. Chen, Y. Chen, J. Chu, and X. Xu, Bridged bowtie aperture antenna for producing an electromagnetic hot spot, ACS Photonics 4, 567 (2017).

[14] R. M. Bakker, D. Permyakov, Y. F. Yu, D. Markovich, R. Paniagua-Domínguez, L. Gonzaga, A. Samusev, 
Y. Kivshar, B. Luk'yanchuk, and A. I. Kuznetsov, Magnetic and electric hotspots with silicon nanodimers, Nano Lett. 15, 2137 (2015).

[15] T. Itoh, Y. S. Yamamoto, Y. Kitahama, and J. Balachandran, One-dimensional plasmonic hotspots located between silver nanowire dimers evaluated by surface-enhanced resonance Raman scattering, Phys. Rev. B 95, 115441 (2017).

[16] Y. Tian, Z. Shuai, J. Shen, L. Zhang, S. Chen, C. Song, B. Zhao, Q. Fan, and L. Wang, Plasmonic heterodimers with binding site-dependent hot spot for surface-enhanced Raman scattering, Small 14, 1800669 (2018).

[17] T.-H. Yang, Y.-W. Harn, M.-Y. Pan, L.-D. Huang, M.-C. Chen, B.-Y. Li, P.-H. Liu, P.-Y. Chen, C.-C. Lin, P.-K. Wei, L.-J. Chen, and J.-M. Wu, Ultrahigh density plasmonic hot spots with ultrahigh electromagnetic field for improved photocatalytic activities, Appl. Catal. B 181, 612 (2016).

[18] A. Kinkhabwala, Z. Yu, S. Fan, Y. Avlasevich, K. Müllen, and W. E. Moerner, Large single-molecule fluorescence enhancements produced by a bowtie nanoantenna, Nat. Photonics 3, 654 (2009).

[19] A. Blank, E. Dikarov, R. Shklyar, and Y. Twig, Inductiondetection electron spin resonance with sensitivity of 1000 spins: En route to scalable quantum computations, Phys. Lett. A 377, 1937 (2013).

[20] M. Kasperczyk, S. Person, D. Ananias, L. D. Carlos, and L. Novotny, Excitation of Magnetic Dipole Transitions at Optical Frequencies, Phys. Rev. Lett. 114, 163903 (2015).

[21] A. V. Malakhovskii, S. L. Gnatchenko, I. S. Kachur, V. G. Piryatinskaya, and I. A. Gudim, Transformation of the $\mathrm{HoFe}_{3}\left(\mathrm{BO}_{3}\right)_{4}$ absorption spectra at reorientation magnetic transitions and local properties in the excited ${ }^{5} F_{5}$ states of the $\mathrm{Ho}^{3}+$ ion, Phys. Rev. B 96, 224430 (2017).

[22] M. L. Juan, M. Righini, and R. Quidant, Plasmon nanooptical tweezers, Nat. Photonics 5, 349 (2011).

[23] D. R. Smith, J. B. Pendry, and M. C. K. Wiltshire, Metamaterials and negative refractive index, Science 305, 788 (2004).

[24] M. W. Klein, C. Enkrich, M. Wegener, and S. Linden, Second-harmonic generation from magnetic metamaterials, Science 313, 502 (2006).

[25] K. Imura, H. Okamoto, M. K. Hossain, and M. Kitajima, Visualization of localized intense optical fields in single gold-nanoparticle assemblies and ultrasensitive Raman active sites, Nano Lett. 6, 2173 (2006).

[26] P. Kusch, S. Mastel, N. S. Mueller, N. Morquillas Azpiazu, S. Heeg, R. Gorbachev, F. Schedin, U. Hübner, J. I. Pascual, S. Reich, and R. Hillenbrand, Dual-scattering nearfield microscope for correlative nanoimaging of SERS and electromagnetic hotspots, Nano Lett. 17, 2667 (2017).

[27] N. Caselli, F. La China, W. Bao, F. Riboli, A. Gerardino, L. Li, E. H. Linfield, F. Pagliano, A. Fiore, P. J. Schuck, S. Cabrini, A. Weber-Bargioni, M. Gurioli, and F. Intonti, Deep-subwavelength imaging of both electric and magnetic localized optical fields by plasmonic campanile nanoantenna, Sci. Rep. 5, 9606 (2015).

[28] P. Ghenuche, S. Cherukulappurath, T. H. Taminiau, N. F. van Hulst, and R. Quidant, Spectroscopic Mode Mapping of Resonant Plasmon Nanoantennas, Phys. Rev. Lett. 101, 116805 (2008).

[29] B. Lahiri, G. Holland, V. Aksyuk, and A. Centrone, Nanoscale imaging of plasmonic hot spots and dark modes with the photothermal-induced resonance technique, Nano Lett. 13, 3218 (2013).

[30] S. Dodson, M. Haggui, R. Bachelot, J. Plain, S. Li, and Q. Xiong, Optimizing electromagnetic hotspots in plasmonic bowtie nanoantennae, J. Phys. Chem. Lett. 4, 496 (2013).

[31] K. Schraml, M. Spiegl, M. Kammerlocher, G. Bracher, J. Bartl, T. Campbell, J. J. Finley, and M. Kaniber, Optical properties and interparticle coupling of plasmonic bowtie nanoantennas on a semiconducting substrate, Phys. Rev. B 90, 035435 (2014).

[32] S. Sederberg and A. Y. Elezzabi, Nanoscale plasmonic contour bowtie antenna operating in the mid-infrared, Opt. Express 19, 15532 (2011).

[33] B. J. Roxworthy, K. D. Ko, A. Kumar, K. H. Fung, E. K. C. Chow, G. L. Liu, N. X. Fang, and K. C. Toussaint, Application of plasmonic bowtie nanoantenna arrays for optical trapping, stacking, and sorting, Nano Lett. 12, 796 (2012).

[34] H. Guo, B. Simpkins, J. D. Caldwell, and J. Guo, Resonance spectra of diabolo optical antenna arrays, AIP Adv. 5, 107149 (2015).

[35] V. Křápek, A. Konečná, M. Horák, F. Ligmajer, M. Stöger-Pollach, M. Hrtoň, J. Babocký, and T. Šikola, Independent engineering of individual plasmon modes in plasmonic dimers with conductive and capacitive coupling, Nanophotonics 9, 623 (2020).

[36] S. Cakmakyapan, N. A. Cinel, A. O. Cakmak, and E. Ozbay, Validation of electromagnetic field enhancement in near-infrared through Sierpinski fractal nanoantennas, Opt. Express 22, 19504 (2014).

[37] F. Falcone, T. Lopetegi, M. A. G. Laso, J. D. Baena, J. Bonache, M. Beruete, R. Marqués, F. Martín, and M. Sorolla, Babinet Principle Applied to the Design of Metasurfaces and Metamaterials, Phys. Rev. Lett. 93, 197401 (2004).

[38] M. Hentschel, T. Weiss, S. Bagheri, and H. Giessen, Babinet to the half: Coupling of solid and inverse plasmonic structures, Nano Lett. 13, 4428 (2013).

[39] A. Bitzer, A. Ortner, H. Merbold, T. Feurer, and M. Walther, Terahertz near-field microscopy of complementary planar metamaterials: Babinet's principle, Opt. Express 19, 2537 (2011).

[40] T. Zentgraf, T. P. Meyrath, A. Seidel, S. Kaiser, H. Giessen, C. Rockstuhl, and F. Lederer, Babinet's principle for optical frequency metamaterials and nanoantennas, Phys. Rev. B 76, 033407 (2007).

[41] H. Mizobata, K. Ueno, H. Misawa, H. Okamoto, and K. Imura, Near-field spectroscopic properties of complementary gold nanostructures: Applicability of Babinet's principle in the optical region, Opt. Express 25, 5279 (2017).

[42] M. Horák, V. Křápek, M. Hrtoň, A. Konečná, F. Ligmajer, M. Stöger-Pollach, T. Šamořil, A. Paták, Z. Édes, O. Metelka, J. Babocký, and T. Šikola, Limits of Babinet's principle for solid and hollow plasmonic antennas, Sci. Rep. 9, 4004 (2019).

[43] P. B. Johnson and R. W. Christy, Optical constants of the noble metals, Phys. Rev. B 6, 4370 (1972).

[44] J. A. Scholl, A. García-Etxarri, A. L. Koh, and J. A. Dionne, Observation of quantum tunneling between two plasmonic nanoparticles, Nano Lett. 13, 564 (2013). 
[45] M. Mivelle, I. Ibrahim, F. Baida, G. Burr, D. Nedeljkovic, D. Charraut, J.-Y. Rauch, R. Salut, and T. Grosjean, Bowtie nano-aperture as interface between near-fields and a singlemode fiber, Opt. Express 18, 15964 (2010).

[46] J. Zhou, T. Koschny, M. Kafesaki, E. N. Economou, J. B. Pendry, and C. M. Soukoulis, Saturation of the Magnetic Response of Split-Ring Resonators at Optical Frequencies, Phys. Rev. Lett. 95, 223902 (2005).

[47] E. M. Purcell, Spontaneous emission probabilities at radio frequencies, Phys. Rev. 69, 681 (1946).

[48] H. A. Atwater and A. Polman, Plasmonics for improved photovoltaic devices, Nat. Mater. 9, 205 (2010).

[49] Z. Édes, V. Křápek, and T. Šikola, Modeling of plasmonenhanced photoluminescence of Si nanocrystals embedded in thin silicon-rich oxinitride layer, Acta Phys. Pol. A 129, A70 (2016).

[50] E. Palacios, S. Park, S. Butun, L. Lauhon, and K. Aydin, Enhanced radiative emission from monolayer MoS2 films using a single plasmonic dimer nanoantenna, Appl. Phys. Lett. 111, 031101 (2017).

[51] M. Eggleston, S. Desai, K. Messer, S. Madhvapathy, J. Xiao, X. Zhang, E. Yablonovitch, A. Javey, and M. C. Wu, in CLEO: 2015 (Optical Society of America, Washington, 2015), p. FTu1E.5.

[52] D. Gao, W. Ding, M. Nieto-Vesperinas, X. Ding, M. Rahman, T. Zhang, C. Lim, and C.-W. Qiu, Optical manipulation from the microscale to the nanoscale: Fundamentals, advances and prospects, Light: Sci. Appl. 6, e17039 (2017).

[53] L. Novotny, R. X. Bian, and X. S. Xie, Theory of Nanometric Optical Tweezers, Phys. Rev. Lett. 79, 645 (1997).

[54] A. S. Baburin, A. M. Merzlikin, A. V. Baryshev, I. A. Ryzhikov, Y. V. Panfilov, and I. A. Rodionov, Silver-based plasmonics: Golden material platform and application challenges [invited], Opt. Mater. Express 9, 611 (2019).
[55] G. V. Naik, J. Kim, and A. Boltasseva, Oxides and nitrides as alternative plasmonic materials in the optical range [invited], Opt. Mater. Express 1, 1090 (2011).

[56] See Supplemental Material at http://link.aps.org/supple mental/10.1103/PhysRevApplied.13.054045 for silver and ZrN plasmonic antennas.

[57] S. V. Boriskina, T. A. Cooper, L. Zeng, G. Ni, J. K. Tong, Y. Tsurimaki, Y. Huang, L. Meroueh, G. Mahan, and G. Chen, Losses in plasmonics: From mitigating energy dissipation to embracing loss-enabled functionalities, Adv. Opt. Photon. 9, 775 (2017).

[58] P. Yu, S. Chen, J. Li, H. Cheng, Z. Li, and J. Tian, Coenhancing and -confining the electric and magnetic fields of the broken-nanoring and the composite nanoring by azimuthally polarized excitation, Opt. Express 21, 20611 (2013).

[59] B. J. Roxworthy and K. C. Toussaint, Simultaneously tuning the electric and magnetic plasmonic response using capped bi-metallic nanoantennas, Nanoscale 6, 2270 (2014).

[60] M. Kerker, D.-S. Wang, and C. L. Giles, Electromagnetic scattering by magnetic spheres, J. Opt. Soc. Am. 73, 765 (1983).

[61] J. Zhang, M. ElKabbash, R. Wei, S. C. Singh, B. Lam, and C. Guo, Plasmonic metasurfaces with $42.3 \%$ transmission efficiency in the visible, Light Sci. Appl. 8, 53 (2019).

[62] G. Yoon, I. Kim, S. So, J. Mun, M. Kim, and J. Rho, Fabrication of three-dimensional suspende, interlayered and hierarchical nanostructures by accuracy-improved electron beam lithography overlayd, Sci. Rep. 7, 6668 (2017).

[63] N. Liu, H. Guo, L. Fu, S. Kaiser, H. Schweizer, and H. Giessen, Three-dimensional photonic metamaterials at optical frequencies, Nat. Mater. 7, 31 (2007). 